\title{
Global Analysis of Beddington-DeAngelis Type Chemostat Model with Nutrient Recycling and Impulsive Input
}

\author{
Mehbuba Rehim, Lingling Sun, Ahmadjan Muhammadhaji* \\ College of Mathematics and System Sciences, Xinjiang University, Urumqi, China \\ Email: *ahmadjanm@gmail.com
}

Received June 6, 2013; revised July 6, 2013; accepted July 13, 2013

Copyright (C) 2013 Mehbuba Rehim et al. This is an open access article distributed under the Creative Commons Attribution License, which permits unrestricted use, distribution, and reproduction in any medium, provided the original work is properly cited.

\begin{abstract}
In this paper, a Beddington-DeAngelis type chemostat model with nutrient recycling and impulsive input is considered. Except using Floquet theorem, introducing a new method combining with comparison theorem of impulse differential equation and by using the Liapunov function method, the sufficient and necessary conditions on the permanence and extinction of the microorganism are obtained. Two examples are given in the last section to verify our mathematical results. The numerical analysis shows that if only the system is permanent, then it also is globally attractive.
\end{abstract}

Keywords: Beddington-DeAngelis Model; Chemostat Model; Nutrient Recycling; Global Attractivity

\section{Introduction}

The chemostat is an important and basic laboratory apparatus for culturing microorganisms. It can be used to investigate microbial growth and has the advantage that parameters are easily measurable. The chemostat plays an important role in bioprocessing, hence the model has been studied by more and more people. Chemostats with periodic inputs were studied [1,2], those with periodic washout rate [3,4], and those with periodic input and washout [5]. In recent years, those with nutrient recycling [6-10] have been investigated and some investing results were obtained. Now many scholars pointed out that it was necessary to consider models with periodic perturbations, since those phenomena might be exposed in many real words. However, there are some other perturbations such as floods, fires and drainaye of sewage which are not suitable to be considered continually. Those perturbations bring sudden changes to the system. Systems with sudden changes are involving in impulsive differential equations which have been studied intensively and systematically [11-13]. Impulsive differential equations are found in almost every domain of applied sciences.

Recently, many papers studied chemostat model with impulsive effect the Lotka-Volterra type or Monod type functional response. But there are few papers which study a chemostat model with Beddington-DeAngelis

\footnotetext{
"Corresponding author.
}

functional response, especially a Beddinton-DeAngelis type chemostat with nutrient recycling. The BeddingtonDeAngelis functional response is introduced by Beddington and DeAngelis $[14,15]$. It is similar to the wellknown Holling II functional response but has an extra term $B(t)$ in the denominator that models mutual interference in species. The model, we consider in this paper, takes the form:

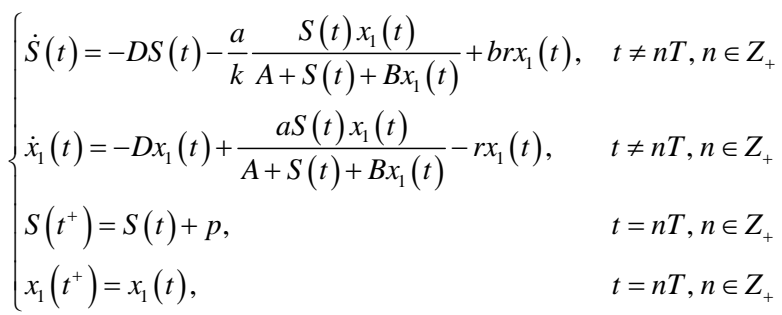

where $S(t), x_{1}(t)$ represent the concentration of limiting substrate and the microorganism respectively, $D$ is the dilution rate, $a$ is the uptake constant of the microorganism, $k$ is the yield of the microorganism $x_{1}(t)$ per unit mass of substrate, $r$ is the death rate of microorganism, $b$ is the fraction of the nutrient recycled by bacterial decomposition of the dead microorganism, $p$ is the amount of limiting substrate pulsed each $T, T$ is the period of pulsing. Obviously, we have $0 \leq b \leq 1$ and $0 \leq k \leq 1$. $D, A, B, k, a, p$ are all positive constants.

The organization of this paper is as the following. In Section 2, we introduce some useful notations and lem- 
mas. In Section 3, we will state and prove the main results on the global asymptotic stability and permanence. In Section 4, we give a brief discussion and the numerical analysis.

\section{Preliminaries}

In this section, we will give some notations and lemmas which will be used for our main results. Firstly, for convenience, we set $x(t)=\frac{x_{1}(t)}{k}$, then system (1) becomes

$$
\begin{cases}\dot{S}(t)=-D S(t)-\frac{a S(t) x(t)}{A+S(t)+B k x(t)}+b r k x(t), & t \neq n T, n \in Z_{+} \\ \dot{x}(t)=-D x(t)+\frac{a S(t) x(t)}{A+S(t)+B x(t)}-r x(t), & t \neq n T, n \in Z_{+} \\ S\left(t^{+}\right)=S(t)+p, & t=n T, n \in Z_{+} \\ x\left(t^{+}\right)=x(t), & t=n T, n \in Z_{+}\end{cases}
$$

Let $R_{+}=[0, \infty), R_{+}^{2}=\left\{X \in R^{2}, X=(S, x)\right\}$. $S\left(n T^{+}\right)=\lim _{t \rightarrow n T^{+}} S(t), x\left(n T^{+}\right)=\lim _{t \rightarrow n T^{+}} x(t)$, $S(t)$ is left continuous at $t=n T$ and $x(t)$ is continuous at $t=n T$.

Lemma 1. Suppose $(S(t) ; x(t))$ is any solution of system (2) with initial solution $S\left(0^{+}\right)>0, x\left(0^{+}\right)>0$. Then $(S(t), x(t)) \geq 0$ for all $t \geq 0$. Moreover, if $\left(S\left(0^{+}\right), x\left(0^{+}\right)\right)>0$, then $(S(t), x(t))>0$ for all $t \geq 0$.

The proof of Lemma 1 is simple, we omit it here.

In what follows, we give some basic properties about the following system.

$$
\begin{cases}\dot{u}(t)=-D u(t), & t \neq n T, n \in Z_{+} \\ u\left(t^{+}\right)=u(t)+P, & t=n T, n \in Z_{+}\end{cases}
$$

Clearly,

$$
\begin{gathered}
u^{*}(t)=\frac{p \exp (-D(t-n T))}{1-\exp (-D T)}, t \in(n T,(n+1) T], n \in Z_{+}, \\
\left(u^{*}(0)=\frac{p}{1-\exp (-D T)}\right)
\end{gathered}
$$

is a positive periodic solution of system (3). Any solution of system (3) is

$$
\begin{aligned}
& u(t)=\left[u\left(0^{+}\right)-u^{*}\left(0^{+}\right)\right] \exp (-D t)+u^{*}(t), \\
& t \in(n T,(n+1) T], n \in Z_{+} .
\end{aligned}
$$

Hence, we have the following result.

Lemma 2. System (3) has a positive periodic solution $u^{*}(t)$ and $\left|u(t)-u^{*}(t)\right| \rightarrow 0$, as $t \rightarrow \infty$ for any solution $u(t)$ of system (3). Moreover, $u(t) \geq u^{*}(t)$ if $u\left(0^{+}\right) \geq u^{*}\left(0^{+}\right)$and $u(t) \leq u^{*}(t)$ and $u\left(0^{+}\right) \leq u^{*}\left(0^{+}\right)$.

The proof of Lemma 2 can be found in [16].

Lemma 3. There exists a constant $M>0$ such that $S(t)$ $<M, x(t)<M$ for each solution of $(S(t) ; x(t))$ system (2), for $t$ large enough.

Proof Let $(S(t) ; x(t))$ be any solution of system (2) with initial value $\left(S\left(0^{+}\right), x\left(0^{+}\right)\right) \in R_{+}^{2}$. Define a function $V(t)=S(t)+x(t)$.

Then

$$
\left\{\begin{array}{rlrl}
\dot{V}(t) & =-D(S(t)+x(t))+(b k r-r) x(t) & \\
& =-D V(t)+r(b k-1) x(t) & & \\
& \leq-D V(t), & & t \neq n T, n \in Z_{+} \\
V\left(t^{+}\right) & =V(t)+p, & & t=n T, n \in Z_{+}
\end{array}\right.
$$

From the comparison theorem of impulsive differential equations, we have $V(t) \cdot u(t)$ for all $t, 0$, where $u(t)$ is the solution of system (3). From Lemma 2, we have $V(t) \leq u(t) \rightarrow u^{*}(t)$ as $t \rightarrow \infty$, where

$$
\begin{aligned}
& u^{*}(t)=\frac{p \exp (-D(t-n T))}{1-\exp (-D T)} \\
& \text { for all } t \in(n T,(n+1) T], n \in Z_{+} .
\end{aligned}
$$

Hence,

$$
\lim _{x \rightarrow \infty} V(t) \leq \lim _{x \rightarrow \infty} u^{*}(t) \leq \frac{p}{1-\exp (-D T)} .
$$

Thus, $V(t)$ is ultimately bounded. From the definition of $V(t)$, there exists a constant

$$
M=\frac{p}{1-\exp (-D T)}
$$

such that $S(t)<M, x(t)<M$ for any solution $(S(t), x(t))$ of system (2), for $t$ large enough. This completes the proof.

The solution of system (2) corresponding to $x(t)=0$ is called microorganism-free periodic solution. For system (2), if we choose $x(t) \equiv 0$, then system (2) becomes to the following system

$$
\begin{cases}\dot{S}(t)=-D S(t), & t \neq n T, n \in Z_{+} \\ S\left(t^{+}\right)=S(t)+p, & t=n T, n \in Z_{+}\end{cases}
$$

System (4) has a unique global uniformly attractive positive solution

$$
S^{*}(t)=u^{*}(t)=\frac{p \exp (-D(t-n T))}{1-\exp (-D T)}
$$

Hence, system (2) has a positive periodic solution $\left(u^{*}(t), 0\right)$ at which microorganism culture fails. In the 
next section, we will study the global asymptotical stability of the microorganism-free periodic solution $\left(u^{*}(t), 0\right)$ as a solution of system (2).

\section{Main Results}

\section{Theorem 1. Suppose}

$$
\int_{0}^{T}\left[\frac{a u^{*}(t)}{A+u^{*}(t)}-(D+r)\right] \mathrm{d} t \leq 0
$$

Then periodic solution $\left(u^{*}(t), 0\right)$ of system (2) is globally attractive.

Proof Let $(S(t), x(t))$ be any positive solution of system (2). Define a function as follows

$$
V(t)=S(t)+x(t)
$$

Then similar to the proof of Lemma 3, we obtain $V(t) \leq u(t)$ for all $t \geq 0$, where $u(t)$ is the solution of system (3) and $u(t) \rightarrow u^{*}(t)$ as $t \rightarrow \infty$. Hence, there exists a function $a(t): R_{+} \rightarrow R$ satisfying $a(t) \rightarrow 0$ as $t \rightarrow \infty$ such that

$$
V(t) \leq u(t)=u^{*}(t)+a(t) \text {. for all } t \geq 0 .
$$

By the definition of $V(t)$, we have

$$
S(t) \leq u^{*}(t)+a(t)-x(t) .
$$

It follows from the second equation of system (2) that

$$
\dot{x}(t) \leq x(t)\left(\frac{a\left(u^{*}(t)+\alpha(t)-x(t)\right)}{A+u^{*}(t)+\alpha(t)-x(t)+B k x(t)}-(D+r)\right) \leq x(t)\left(\frac{a\left(u^{*}(t)+\alpha(t)-x(t)\right)}{A+u^{*}(t)+\alpha(t)-x(t)}-(D+r)\right) .
$$

From condition (5), for any enough small $\varepsilon_{0}>0$ we have

$$
\begin{aligned}
& \lim _{x \rightarrow \infty}\left(\int_{t}^{t+T} \frac{a\left(u^{*}(t)+\alpha(t)-\varepsilon_{0}\right)}{A+u^{*}(t)+\alpha(t)-\varepsilon_{0}} \mathrm{~d} t-(D+r) T\right) \\
& =\int_{0}^{T}\left(\frac{a\left(u^{*}(t)-\varepsilon_{0}\right)}{A+u^{*}(t)-\varepsilon_{0}}\right) \mathrm{d} t-(D+r) T \\
& <0
\end{aligned}
$$$$
\int_{0}^{T}\left(\frac{a\left(u^{*}(t)-\varepsilon_{0}\right)}{A+u^{*}(t)-\varepsilon_{0}}\right) \mathrm{d} t-(D+r) T<0 .
$$

Since $\lim _{x \rightarrow \infty} a(t)=0$, which gives

Hence, there exist constants $\eta>0$ and $T_{0}>0$, such that

$$
\int_{t}^{t+T} \frac{a\left(u^{*}(t)+\alpha(t)-\varepsilon_{0}\right)}{A+u^{*}(t)+\alpha(t)-\varepsilon_{0}} \mathrm{~d} t-(D+r) T \leq-\eta \text { for } t>T_{0} \text { and }|a(t)|<1 .
$$

If $x(t) \geq \varepsilon_{0}$ for all $t \geq T_{0}$, then from (6) we have

$$
\begin{gathered}
\left.\dot{x}(t) \leq x(t)\left(\frac{a\left(u^{*}(t)+\alpha(t)-\varepsilon_{0}\right)}{A+u^{*}(t)+\alpha(t)-\varepsilon_{0}}-(D+r)\right) \cdot \quad \begin{array}{r}
\text { For any } t \geq T_{0}, \text { we choose an } \\
t \in\left[T_{0}, T_{0}+(p+1) T\right), \text { then inte } \\
\text { from (7) we have }
\end{array}\right) \mathrm{d} v \\
x(t) \leq x\left(T_{0}\right) \exp \int_{T_{0}}^{t}\left(\frac{a\left(u^{*}(v)+\alpha(v)-\varepsilon_{0}\right)}{A+u^{*}(v)+\alpha(v)-\varepsilon_{0}}-(D+r)\right) \\
\leq x\left(T_{0}\right) \exp \left(\int_{T_{0}}^{T_{0}+p T}+\int_{T_{0}+p T}^{t}\right)\left(\frac{a\left(u^{*}(v)+\alpha(v)-\varepsilon_{0}\right)}{A+u^{*}(v)+\alpha(v)-\varepsilon_{0}}-(D+r)\right) \mathrm{d} v \\
\leq x\left(T_{0}\right) \exp (-\eta p) \exp \int_{T_{0}+p T_{0}}^{t}\left(\frac{a\left(u^{*}(v)+\alpha(v)-\varepsilon_{0}\right)}{A+u^{*}(v)+\alpha(v)-\varepsilon_{0}}-(D+r)\right) \mathrm{d} v \\
\leq x\left(T_{0}\right) \exp (-\eta p) \exp \left(\sigma_{0} T\right),
\end{gathered}
$$

For any $t \geq T_{0}$, we choose an integer $P \geq 0$ such that $t \in\left[T_{0}, T_{0}+(p+1) T\right)$, then integrating (8) from $T_{0}$ to $t$,

where $\sigma_{0}=\frac{a\left(M+1-\varepsilon_{0}\right)}{A+M+1-\varepsilon_{0}}-(D+r)$ and $M$ is given in

Lemma 3. Since $p \rightarrow \infty$ as $t \rightarrow \infty$, from (9) we have $x(0) \rightarrow 0$ as $t \rightarrow \infty$, which is a contradiction. Hence, there is a $t^{*} \geq T_{0}, T_{0}$, such that $x\left(t^{*}\right) \leq \varepsilon_{0}$.

Now, we claim that there exists a constant $M_{0}>1$ 
such that

$$
x(t) \leq \varepsilon_{0} M_{0} \text { for all } t \geq t^{*}
$$

In fact, if there exists a $t_{1} \geq t^{*}$ such that $x\left(t_{1}\right)>\varepsilon_{0} M_{0}$, then there exists a $t_{2} \in\left(t^{*}, t_{1}\right)$ such that $x\left(t_{2}\right)=\varepsilon_{0}$ and $x(t)>\varepsilon_{0}$ for $t_{2} \in\left(t_{2}, t_{1}\right)$. Choose an integer $p \geq 0$ such that $t_{1} \in\left[t_{2}+p T, t_{2}+(p+1) T\right]$. Since for any $t_{2} \in\left(t_{2}, t_{1}\right)$

$$
\dot{x}(t) \leq x(t)\left(\frac{a\left(u^{*}(t)+\alpha(t)-\varepsilon_{0}\right)}{A+u^{*}(t)+\alpha(t)-\varepsilon_{0}}-(D+r)\right),
$$

integrating the above inequality from $t_{2}$ to $t_{1}$, from (7) we obtain (10).

Obviously, let $M_{0}=\exp \left(\sigma_{0} T\right)$, then from (10) we obtain a contradiction. Hence, $x(t) \leq \varepsilon_{0} M_{0}$ for all $t \geq t^{*}$. Since $\varepsilon_{0}$ is arbitrary, we finally have $\lim _{x \rightarrow \infty} x(t)=0$.

This completes the proof.

\section{Theorem 2. Suppose}

$$
\int_{0}^{T}\left[\frac{a u^{*}(t)}{A+u^{*}(t)}-(D+r)\right] \mathrm{d} t>0 .
$$

Then system (2) is permanent.

Proof Let $(S(t) ; x(t))$ be any solution of system (2) with initial value $\left(S\left(0^{+}\right), x\left(0^{+}\right)\right) \in R_{+}^{2}$. By Lemma 3 , the first equation of system (2) becomes

$$
\left\{\begin{array}{rlrl}
\dot{S}(t) & \geq-D S(t)-\frac{a S(t) x(t)}{A+S(t)+B k x(t)} & \\
& \geq-D S(t)-\frac{a M S(t)}{A} & \\
& \leq-\left(D+\frac{a M}{A}\right) S(t), & t \neq n T, n \in Z_{+} \\
S\left(t^{+}\right) & =S(t)+p, & t & =n T, n \in Z_{+} .
\end{array}\right.
$$

Using Lemma 2 and the comparison theorem of impulsive differential equation, we obtain $S(t) \geq v(t)$ for all, $t \geq 0$ where $v(t)$ is the solution of the following impulsive system

$$
\begin{cases}\dot{v}(t)=-\left(D+\frac{a M}{A}\right) v(t), & t \neq n T, n \in Z_{+} \\ v\left(t^{+}\right)=v(t)+p, & t=n T, n \in Z_{+} .\end{cases}
$$

with initial condition $v\left(0^{+}\right)=S\left(0^{+}\right)$. Further from Lemma 2, we have

$$
\lim _{x \rightarrow \infty} v(t)=v^{*}(t)
$$

where

$$
v^{*}(t)=\frac{p \exp \left(-\left(D+\frac{a M}{A}\right)(t-n T)\right)}{1-\exp \left(-\left(D+\frac{a M}{A}\right) T\right)} .
$$

Therefore, we finally obtain

$$
\begin{aligned}
\liminf _{x \rightarrow \infty} S(t) & \geq \liminf _{x \rightarrow \infty} v(t)=\liminf _{x \rightarrow \infty} v^{*}(t) \\
& \geq \frac{p \exp \left(-\left(D+\frac{a M}{A}\right) T\right)}{1-\exp \left(-\left(D+\frac{a M}{A}\right) T\right)}
\end{aligned}
$$

This shows that $S(t)$ in system (2) is permanent.

In the following, we want to find a constant $m_{2}$, such that $x(t)>m_{2}$ for $t$ large enough.

Since

$$
\int_{0}^{T}\left[\frac{a u^{*}(t)}{A+u^{*}(t)}-(D+r)\right] \mathrm{d} t>0,
$$

we can chose a constant $\varepsilon_{2}>0$ small enough such that

$$
\sigma=\int_{0}^{T}\left[\frac{a\left(u^{*}(t)-\varepsilon_{2}\right)}{A+u^{*}(t)-\varepsilon_{2}+B k \varepsilon_{2}}-(D+r)\right] \mathrm{d} t>0 .
$$

Consider the following auxiliary impulsive system

$$
\begin{cases}\dot{y}(t)=-\left(D+\frac{a m_{3}}{A}\right) y(t), & t \neq n T, n \in Z_{+} \\ y\left(t^{+}\right)=y(t)+p, & t=n T, n \in Z_{+} .\end{cases}
$$

$$
\begin{aligned}
x(t) & \leq x\left(t_{2}\right) \exp \int_{t_{2}}^{t_{1}}\left(\frac{a\left(u^{*}(t)+\alpha(t)-\varepsilon_{0}\right)}{A+u^{*}(t)+\alpha(t)-\varepsilon_{0}}-(D+r)\right) \mathrm{d} t \\
& \leq x\left(t_{2}\right) \exp \left(\int_{t_{2}}^{t_{2}+p T}+\int_{t_{2}+p T}^{t_{1}}\right)\left(\frac{a\left(u^{*}(t)+\alpha(t)-\varepsilon_{0}\right)}{A+u^{*}(t)+\alpha(t)-\varepsilon_{0}}-(D+r)\right) \mathrm{d} t \\
& \leq x\left(t_{2}\right) \exp (-\eta p) \exp \int_{t_{2}+p T_{0}}^{t_{1}}\left(\frac{a\left(u^{*}(t)+\alpha(t)-\varepsilon_{0}\right)}{A+u^{*}(t)+\alpha(t)-\varepsilon_{0}}-(D+r)\right) \mathrm{d} t \\
& \leq \varepsilon_{0} \exp \left(\sigma_{0} T\right) .
\end{aligned}
$$


from Lemma 2, system (12) has a globally uniformly attractive positive periodic solution

$$
y^{*}(t)=\frac{p \exp \left(-\left(D+\frac{a M}{A}\right)(t-n T)\right)}{1-\exp \left(-\left(D+\frac{a M}{A}\right) T\right)} \text {, for all } t \in(n T,(n+1) T], n \in Z_{+}
$$

Since $\lim _{m_{3} \rightarrow 0} y^{*}(t)=u^{*}(t)$, for above $\varepsilon_{2}>0$, there is a $m_{3}>0$ and $m_{3} \leq \varepsilon_{2}$ such that

$$
y^{*}(t) \geq u^{*}-\frac{\varepsilon_{2}}{2} \text { for } t \geq 0
$$

Further, for above $\varepsilon_{2}>0$ and $M>0$, where $M$ is given in Lemma 3, there is a $T_{0}=T_{0}\left(\varepsilon_{2}, M\right)$ such that for any $t_{0} \geq 0$ and $0 \leq y_{0} \leq M$ we have

$$
\left|y\left(t, t_{0}, y_{0}\right)-y^{*}(t)\right|<\frac{\varepsilon_{2}}{2} \text { for all } t \geq t_{0}+T_{0}
$$

where $y\left(t, t_{0}, y_{0}\right)$ is the solution of system (12) with initial condition $y\left(t_{0}\right)=y_{0}$.

For any $t_{0} \geq 0$, if $x(t) \leq m_{3}$ for all $t \geq t_{0}$, then from system (2) we have

$$
\begin{cases}\dot{S}(t) \geq-\left(D+\frac{a m_{3}}{A}\right) S(t), & t \neq n T, n \in Z_{+} \\ S\left(t^{+}\right)=S(t)+p, & \text { for all } t \geq t_{0}\end{cases}
$$

By the comparison theorem of impulsive differential equations, we have $S(t) \geq y(t)$ for $t \geq t_{0}$, where $y(t)$ is the solution of system (12) with initial condition $y\left(t_{0}^{+}\right)=S\left(t_{0}^{+}\right)$. From (14) we have

$$
\left|y(t)-y^{*}(t)\right|<\frac{\varepsilon_{2}}{2} \text { for all } t \geq t_{0}+T_{0}
$$

Hence, from (13) we further have

$$
S(t) \geq u^{*}(t)-\varepsilon_{2} \text { for all } t \geq t_{0}+T_{0}
$$

From the second equation of system (2) we have

$$
\begin{aligned}
\dot{x}(t) & =x(t)\left(\frac{a S(t)}{A+S(t)+B k x(t)}-(D+r)\right) \geq x(t)\left(\frac{a S(t)}{A+S(t)+B k m_{3}}-(D+r)\right) \\
& \geq x(t)\left(\frac{a\left(u^{*}(t)-\varepsilon_{2}\right)}{A+u^{*}(t)-\varepsilon_{2}+B k \varepsilon_{2}}-(D+r)\right) \text { for all } t \geq t_{0}+T_{0}
\end{aligned}
$$

Let $n_{0} \in Z_{+}$such that $n_{0} \geq t_{0}+T_{0}$. Integrating (15) on $(n T,(n+1) T]$ for all $n \geq n_{0}$, we have

$$
x((n+1) T) \geq x\left(n T^{+}\right) \exp \left(\int_{n T}^{(n+1) T}\left(\frac{a\left(u^{*}(t)-\varepsilon_{2}\right)}{A+u^{*}(t)-\varepsilon_{2}+B k \varepsilon_{2}}-(D+r)\right) \mathrm{d} t\right)=x(n T) \exp (\sigma)
$$

Hence, $x\left(\left(n_{0}+k\right) T\right) \geq x\left(n_{0} T\right) \exp (k \sigma)$ for all $k \geq 0$. Then we have $\lim _{t \rightarrow \infty} x\left(\left(n_{0}+k\right) T\right)=\infty$, which is a contradiction. Hence, there exists a $t_{1} \geq t_{0}+T_{0}$ such that $x\left(t_{1}\right) \geq m_{3}$.

If $x(t) \geq m_{3}$ for all $t \geq t_{1}$, then our goal is obtained. Hence, we need only to consider these solutions which are oscillatory about $m_{3}$. Let $t_{1}$ and $t_{2}$ be two large enough times such that $x\left(t_{1}\right)=x\left(t_{2}\right)=m_{3}$ and $x(t)<m_{3}$ for all $t \in\left(t_{1}, t_{2}\right)$. When $t_{1}-t_{2}<T_{0}$, since

$$
\dot{x}(t) \geq-(D+r) x(t) \text { for all } t \in\left(t_{1}, t_{2}\right)
$$

integrating this inequality for any $t \in\left[t_{1}, t_{2}\right]$, we have

$$
x(t) \geq x\left(t_{1}\right) \exp \int_{t_{1}}^{t}-(D+r) \mathrm{d} v \geq m_{3} \exp \left(-(D+r) T_{0}\right)=m_{2}^{*} .
$$

Let $t_{2}-t_{1}>T_{0}$. For any $t \in\left[t_{1}, t_{2}\right]$, if $t<t_{1}+T_{0}$, then according to the above discussing on the case of $t_{2}-t_{1} \leq T_{0}$, we also have inequality (16). Particularly, we obtain $x\left(t_{1}+T_{0}\right) \geq m_{2}^{*}$, since $x(t) \leq m_{3}$ for all $t \in\left[t_{1}, t_{2}\right]$, from system (2) we have

$$
\begin{cases}\dot{S}(t) \geq-\left(D+\frac{a m_{3}}{A}\right) S(t), & t \neq n T, \quad n \in Z_{+} \\ S\left(t^{+}\right)=S(t)+p, & t=n T, \quad n \in Z_{+} .\end{cases}
$$

Hence, from the comparison theorem of impulsive differential equations, we have $S(t) \geq y(t)$ for all $t \in\left[t_{1}, t_{2}\right]$, where $y(t)$ is the solution of system (12) with initial condition $y\left(t_{1}^{+}\right)=S\left(t_{1}^{+}\right)$. From (14), we have

$$
y(t) \geq y^{*}(t)-\frac{\varepsilon_{2}}{2} \text { for all } t \in\left[t_{1}+T_{0}, t_{2}\right]
$$


Further from (13), we also have

$$
S(t) \geq y(t) \geq y^{*}(t)-\frac{\varepsilon_{2}}{2} \geq U^{*}(t)-\varepsilon_{2} \text { for all } t \in\left[t_{1}+T_{0}, t_{2}\right]
$$

Thus, from system (2), we have

$$
\begin{aligned}
\dot{x}(t) & =x(t)\left(\frac{a S(t)}{A+S(t)+B k x(t)}-(D+r)\right) \geq x(t)\left(\frac{a S(t)}{A+S(t)+B k m_{3}}-(D+r)\right) \\
& \geq x(t)\left(\frac{a\left(u^{*}(t)-\varepsilon_{2}\right)}{A+u^{*}(t)-\varepsilon_{2}+B k \varepsilon_{2}}-(D+r)\right) \text { for all } t \in\left[t_{1}+T_{0}, t_{2}\right]
\end{aligned}
$$

For any $t \in\left[t_{1}+T_{0}, t_{2}\right]$, we choose an integer $p \geq 0$ such that

$$
t \in\left[t_{1}+T_{0}+p T, t_{1}+T_{0}+(p+1) T\right] .
$$

Integrating (17) from $t_{1}+T_{0}$ to $t$, we have

$$
\begin{aligned}
x(t) & =x\left(t_{1}+T_{0}\right) \exp \int_{t_{1}+T_{0}}^{t}\left(\frac{a\left(u^{*}(v)-\varepsilon_{2}\right)}{A+u^{*}(v)-\varepsilon_{2}+B k \varepsilon_{2}}-(D+r)\right) \mathrm{d} v \\
& \leq m_{2}^{*} \exp \left(\int_{t_{1}+T_{0}}^{t_{1}+T_{0}+p T}+\int_{t_{1}+T_{0}+p T}^{t}\right)\left(\frac{a\left(u^{*}(v)-\varepsilon_{2}\right)}{A+u^{*}(v)-\varepsilon_{2}+B k \varepsilon_{2}}-(D+r)\right) \mathrm{d} v \\
& \leq m_{2}^{*} \exp \int_{t_{1}+T_{0}+p T}^{t}\left(\frac{a\left(u^{*}(v)-\varepsilon_{2}\right)}{A+u^{*}(v)-\varepsilon_{2}+B k \varepsilon_{2}}-(D+r)\right) \mathrm{d} v \\
& \leq m_{2}^{*} \exp (-h T) \\
& =: m_{2},
\end{aligned}
$$

where

$$
h=\sup _{t \geq 0}\left(\frac{a\left(u^{*}(t)-\varepsilon_{2}\right)}{A+u^{*}(t)-\varepsilon_{2}+B k \varepsilon_{2}}-(D+r)\right)
$$

From the above discussion, we have $\lim _{t \rightarrow \infty} x(t) \geq m_{2}$, and $m_{2}$ is independent of any solution $(S(t) ; x(t))$ of system (2). This completes the proof.

As a consequence of Theorem 1 and Theorem 2, we have the following corollary.

Corollary 1 For system (2), the following conclusions hold.

a) The microorganism-extinction solution $\left(S^{*}(t), 0\right)$ is globally attractive if and only if

$$
\int_{0}^{T}\left[\frac{a u^{*}(t)}{A+u^{*}(t)}-(D+r)\right] \mathrm{d} t \leq 0 .
$$

b) The microorganism $x(t)$ of System (2) is permanent if and only if

$$
\int_{0}^{T}\left[\frac{a u^{*}(t)}{A+u^{*}(t)}-(D+r)\right] \mathrm{d} t>0 .
$$

\section{Discussion and Numerical Analysis}

In this paper, we investigate Beddington-DeAngelis type

chemostat with nutrient recycling and impulsive input. We prove that the microorganism-free periodic solution of the system (2) is globally attractive. The necessary and sufficient condition for permanence of system (2) are obtained in this paper.

According to Theorem 1, the microorganism-free periodic solution $\left(S^{*}(t), 0\right)$ is globally attractive if (5) hold. That is, this kind of microorganisms can not be cultivated under this condition. Suppose that $r>a-D, a>D$ and set

$$
p^{*}=\frac{A(\exp (D t)-1)\left(\exp \left(\frac{D(D+r) T}{a}\right)-1\right)}{\exp (D t)-\exp \left(\frac{D(D+r) T}{a}\right)} .
$$

Then Theorem 1-2 can be state as: If

$a>D, r>a-D$ and $0<p \leq p^{*}$, then the microorganism will eventually disappear; If $a>D, r>a-D$ and $p>p^{*}$, then system (2) is permanent. This implies that if we choose a smaller impulsive input of nutrient when the death rate of microorganism is larger than some certain value, then the microorganism $x(t)$ will tend to extinct; If we choose a lager impulsive input of nutrient, then system can coexist. By the above analysis, we know 
that conditions for the system coexist or non-coexist are due to the influences of the impulsive perturbations.

In order to illustrate our mathematical results and investigate the effect of impulsive input nutrient we present the following results of a numerical simulation.

From Theorem 1, we consider dynamical behavior of the system (2) with $\mathrm{D}=2, \mathrm{a}=5, \mathrm{~A}=20, \mathrm{~B}=2, \mathrm{~b}=1, \mathrm{k}=$ $0.5, \mathrm{r}=0.5, \mathrm{p}=10, \mathrm{~T}=2$, then system (2) becomes

$$
\begin{cases}\dot{S}(t)=-2 S(t)-\frac{5 S(t) x(t)}{20+S(t)+x(t)}+0.25 x(t), & t \neq 2 n \\ \dot{x}(t)=-2 x(t)+\frac{5 S(t) x(t)}{20+S(t)+B x(t)}-0.5 x(t), & t \neq 2 n \\ S\left(t^{+}\right)=S(t)+10, & t=2 n \\ x\left(t^{+}\right)=x(t), & t=2 n\end{cases}
$$

By calculating, we obtain

$$
S^{*}(t)=\frac{10 \exp (-2(t-2 n))}{1-\exp (-4)}>0, t \in(2 n, 2(n+1)], n \in Z_{+}
$$

and

$$
\int_{0}^{2}\left[\frac{a S^{*}(t)}{A+S^{*}(t)}-(D+r)\right] \mathrm{d} t \leq 5 \ln 0.6687-5<0 .
$$

That is condition (5) holds. We choose initial value $\left(S_{0}, x_{0}\right)=(1,1.3),(1,2.5),(3,3.4),(4,4.7),(5,6),(6,7.3)$, $(7,7.9),(8,9.5),(9,10.7),(10,12.5)$ respectively, then from the numerical simulation (Figure 1) we see that there exists a positive periodic solution $\left(S^{*}(t), 0\right)$ of system (18) such that any solution ( $(\mathrm{S}(\mathrm{t}), x(t))$ of system (20) with initial value $\left(S_{0}, x_{0}\right)$ tends to $\left(S^{*}(t), 0\right)$ as $t \rightarrow \infty$. Therefore, if condition (5) holds, then system (18) has a positive periodic solution which is globally attracttive.

From Theorem 1, we consider dynamical behavior of the system (2) with $\mathrm{D}=1, \mathrm{a}=10, \mathrm{~A}=10, \mathrm{~B}=2, \mathrm{~b}=1, \mathrm{k}$ $=0.5, \mathrm{r}=0.2, \mathrm{p}=12, \mathrm{~T}=2$, then system (2) becomes

$$
\begin{cases}\dot{S}(t)=-S(t)-\frac{10 S(t) x(t)}{10+S(t)+x(t)}+0.2 x(t), & t \neq 2 n \\ \dot{x}(t)=-x(t)+\frac{10 S(t) x(t)}{10+S(t)+B x(t)}-0.2 x(t), & t \neq 2 n \\ S\left(t^{+}\right)=S(t)+12, & t=2 n \\ x\left(t^{+}\right)=x(t), & t=2 n\end{cases}
$$

By calculating, we obtain

$S^{*}(t)=\frac{12 \exp (-(t-2 n))}{1-\exp (-2)}>0, \quad t \in(2 n, 2(n+1)], n \in Z_{+}$

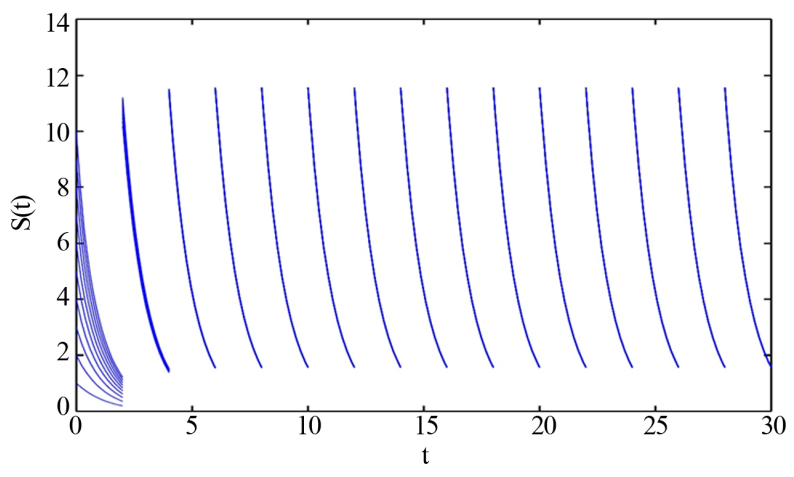

(a)

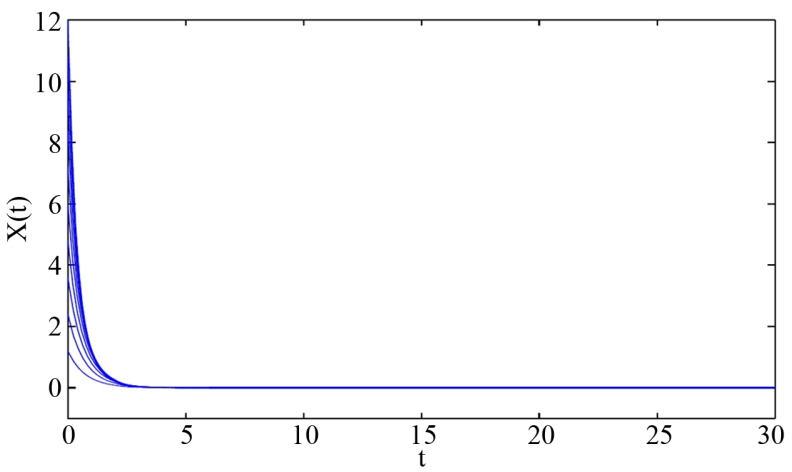

(b)

Figure 1. (a) Time-series of the nutrient $S$ for periodic oscillation; (b) Time-series of the microorganism population $x$ for extinction.

and

$\int_{0}^{2}\left[\frac{a S^{*}(t)}{A+S^{*}(t)}-(D+r)\right] \mathrm{d} t \leq 10 \ln 2.010-2.4 \approx 4.58>0$.

That is condition (11) holds. We choose initial value $\left(S_{0}, x_{0}\right)=(5,2)$, then from the numerical simulation (Figure 2) we see that system (19) is permanent.

It is difficult to study the global attractivity of system (2) analytically. We present here two examples to show that system (2) is global attractive under the condition (11). Setting $\mathrm{D}=1, \mathrm{a}=6, \mathrm{~A}=8, \mathrm{r}=0: 4, \mathrm{p}=18, \mathrm{~T}=2$, b $=1$, so that condition (11) holds. Choosing initial value $\left(S_{0}, x_{0}\right)=(2.5,1.6),(4.7,2.6),(7.1,6.3),(9.4,5.8)$, (12.2,7.3), (14.4), (16.5,9.7), (19.3,11.4), (21.4,12.5), $(23,12)$, respectively, then from the numerical simulation (Figure 3) we see that there exist a unique T-period solution $\left(S^{*}(t), x^{*}(t)\right)$ of system (2) which is globally attractive. Let $\mathrm{D}=1, \mathrm{a}=6, \mathrm{~A}=8, \mathrm{r}=0: 2, \mathrm{p}=20, \mathrm{~T}=2$, $\mathrm{b}$ $=1$. Then the condition (3.9) holds for those parameters. Choosing initial values $\left(S_{0}, x_{0}, c_{0}\right)=(0.5,0.4),(1,0.8)$, $(1.5,1.2),(2,1.6),(2.5,2),(3,2.4),(3.5,2.8),(4,3.2)$, $(4.5,3.6),(5,4)$, respectively, the numerical simulation (Figure 3) also show that system (2) is globally attractive. Therefore, we can guess if only condition (11) holds then 


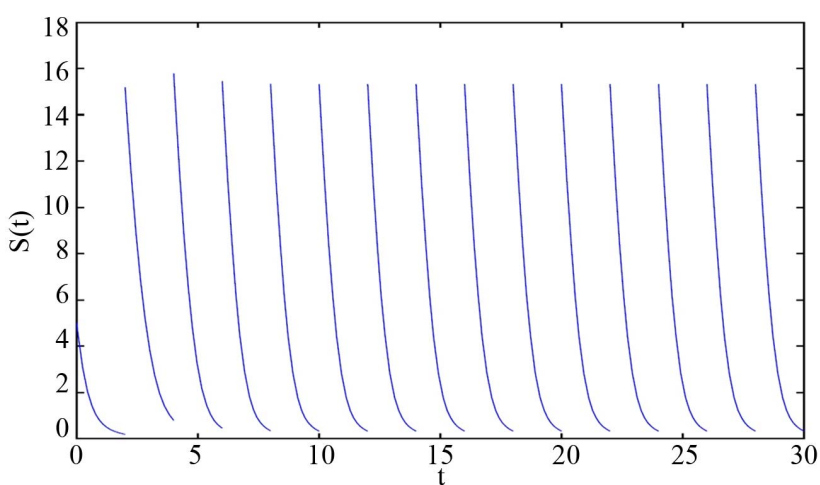

(a)

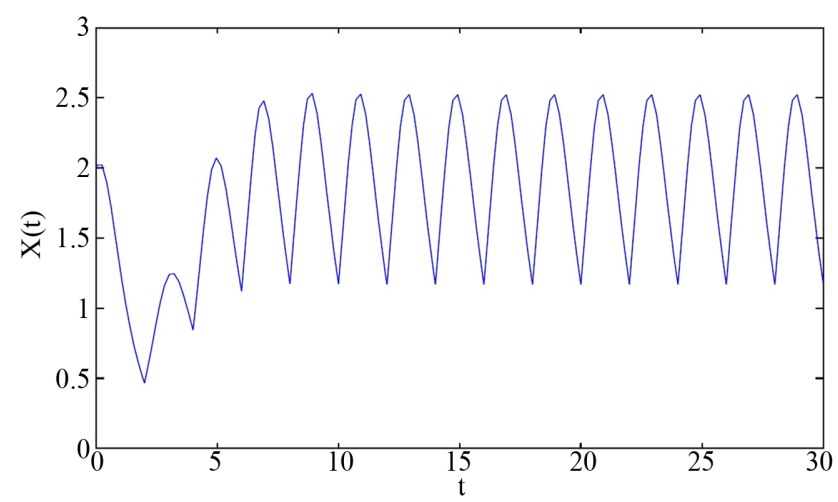

(b)

Figure 2. (a) Time-series of the nutrient $S$ for permanence and periodic oscillation; (b) Time-series of the microorganism population $x$ for permanence.

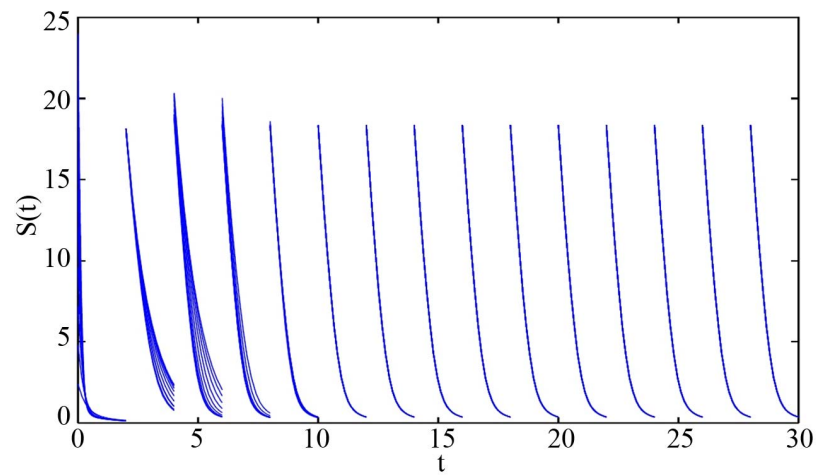

(a)

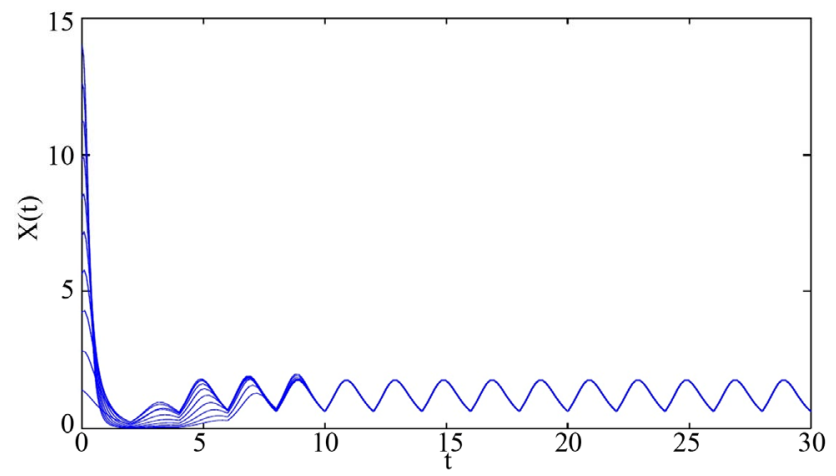

(b)

Figure 3. (a) Time-series of the nutrient $S$ for global attractivity; (b) Time-series of the microorganism population $x$ for global attractivity.

the system (2) has a unique T-period solution which is globally attractive

\section{Acknowledgements}

This work was supported by the National Natural Science Foundation of China (Grant Nos. 11261056, 11261058).

\section{REFERENCES}

[1] H. L. Smith, "Competitive Coexistence in an Oscillating Chemostat," SIAM Journal on Applied Mathematics, Vol. 40, No. 3, 1981, pp. 498-522. doi:10.1137/0140042

[2] S. B. Hsu, “A Competition Model for a Seasonally Fluctuation Nutrient,” Journal of Mathematical Biology, Vol. 9, No. 2, 1980, pp. 115-132.

[3] G. J. Butler, S. B. Hsu and P. Waltman, "A Mathematical Model of the Chemostat with Periodic Washout Rate," SIAM Journal on Applied Mathematics, Vol. 45, No. 3, 1985, pp. 435-449. doi:10.1137/0145025

[4] P. Lemas, "Coexistence of Three Competing Microbial Populations in a Chemostat with Periodic Input and Dilution Rate,” Mathematical Bioscience, Vol. 129, No. 2,
1995, pp. 111-142. doi:10.1016/0025-5564(94)00056-6

[5] S. S. Pilyugin and P. Waltman, "Competition in Unstirred Chemostat with Periodic Input and Washout," SIAM Journal on Applied Mathematics, Vol. 59, No. 2, 1999, pp. 1157-1177.

[6] X. He and S. Ruan, "Global Stability in Chemostat-Type Plankton Models with Delayed Nutrient Recycling,” Research Report, The School of Mathematical and Statistics, University of Sydney, 1996, pp. 96-98.

[7] S. Ruan, "The Effect of Delays on Stability and Persistence in Plankton Models," Nonlinear Analysis: Real Word Applications, Vol. 24, No. 4, 1995, pp. 575-585.

[8] S. Ruan and X. He, "Global Stability in Chemostat-Type Competition Models with Nutrient Recycling," SIAM Journal on Applied Mathematics, Vol. 58, No. 1, 1998, pp. 170-192. doi:10.1137/S0036139996299248

[9] V. S. H. Rao and P. R. S. Rao, "Global Stability in Chemostat Models Involving Time Delays and Wall Growth," Nonlinear Analysis: Real Word Applications, Vol. 5, No. 1, 2005, pp. 141-158.

[10] S. R. J. Jang, "Dynamics of Variable-Yield NutrientPhytoplankton-Zooplankton Models with Nutrient Recycling and Self-Shading," Journal of Mathematical Biology, Vol. 40, No. 3, 2000, pp. 229-250. 
doi:10.1007/s002850050179

[11] S. Sun and L. Chen, "Complex Dynamics of a Chemotat with Variable Yield and Periodically Impulsive Perturbation on the Substrate," Journal of Mathematical Chemistry, Vol. 43, No. 1, 2008, pp. 338-348. doi:10.1007/s10910-006-9200-z

[12] V. Lakshmikantham, D. D. Bainov and P. S. Simeoonov, “Theory of Impulsive Differential Equations," World Science, Singapore City, 1989. doi:10.1142/0906

[13] D. D. Bainov and P. S. Simeoonov, "Impulsive Differential Equations: Periodic Solutions and Applications," Longman Scientific and Thechnical, London, 1993.
[14] D. L. DeAngelis, R. A. Goldstein and R. V. Oneill, “A Model for Trophic Interaction,” Ecology, Vol. 56, No. 4, 1975, pp. 661-692. doi:10.2307/1936298

[15] J. R. Beddington, "Mutual Interference between Parasites and Its Effect on Searching Efficiency," Journal of Animal Ecology, Vol. 44, No. 1, 1975, pp. 331-340. doi:10.2307/3866

[16] Z. D. Teng, R. Gao, R. Mehbuba and K. Wang, “Global Behaviors of Monod type Chemostat Model with Nutrient Recycling and Impusive Onput," Journal of Mathematical Chemistry, Vol. 47, No. 1, 2010, pp. 276-294. doi:10.1007/s10910-009-9567-8 